\title{
Effects of electrode interactions observed in a mechanically controllable break junction
}

\author{
J. Voets and R. J. P. Keijsers \\ Research Institute for Materials, University of Nijmegen, Toernooiveld, 6525 ED Nijmegen, The Netherlands \\ O. I. Shklyarevskii \\ B. Verkin Institute for Low Temperature Physics and Engineering, Ukrainian Academy of Science, \\ 47 Lenin Avenue, 310164 Kharkov, Ukraine \\ H. van Kempen \\ Research Institute for Materials, University of Nijmegen, The Netherlands
}

(Received 18 July 1995)

\begin{abstract}
In this work we will present measurements that show clear features of electrode interactions. Our data have been obtained using a mechanically controllable break junction (MCB). Our findings on strong adhesion forces and anomalous high corrugations are in agreement with recent theory and previous scanning-tunnelingmicroscopy studies. The power of a MCB lies in its high stability, providing the opportunity to observe very subtle effects appearing in the measured tunnel current.
\end{abstract}

\section{INTRODUCTION}

One of the most powerful instruments in physics today is the scanning tunneling microscope (STM). During the past decade it has been widely applied to obtain spectroscopic and topographic information on (semi-)conducting materials. For the usual tip-sample separations of $\sim 10 \AA$, the tunnel current $(I)$ as a function of distance $(z)$ is given by the Tersoff-Haman (TH) approach. ${ }^{1}$ However, if the distance between tip and sample drops below $\sim 7 \AA$, an increasing overlap of the wave functions causes this independent-electrode approximation to break down. Interactions between the two electrodes of the tunnel junction should then be taken into account, showing that the dependence of current $(I)$ on electrode spacing $(z)$ can deviate significantly from the TH approximation. In this paper, we will focus on some of the phenomena encountered at small electrode spacings, say $2 \AA$ $\leq z \leqslant 7 \AA$, using a mechanically controllable break (MCB) junction. $^{2}$

For very small separations $(\leqslant 3 \AA)$ we observed clear indications of strong adhesion forces, as previously reported by Dürig et al., ${ }^{3}$ and recently by Krans et al. ${ }^{4}$ At somewhat larger distances $(3 \AA \leqslant z \leqslant 7 \AA$ ), where the effect of the adhesion forces mentioned above is negligible, we observed another striking effect of electrode interactions. The simple metals we investigated (e.g., $\mathrm{Al}$ and $\mathrm{Au}$ ) showed anomalously high corrugations, which increased as the spacing decreased (see also Ref. 5). Similar observations on laminar materials, such as graphite, have successfully been explained by invoking elastic deformations of the surface due to adhesion forces. This model can, however, not be applied to flat simple metal surfaces, because the effect of adhesion forces is negligible at these separations, as stated above. Starting from a more realistic description of the potential between the electrodes, Tekman and Ciraci derived an effective barrier which varies along the surface. This model turns out to give a rather good explanation for the corrugations observed. ${ }^{6}$

\section{EXPERIMENT}

Our experimental setup is described in detail elsewhere (see Refs. 7 and 8). Thin wires (50-100 $\mu \mathrm{m}$ ) of the sample material were glued to a bending beam (a phosphor bronze plate covered with an insulating layer) at two points separated by $0.3-0.5 \mathrm{~mm}$. The wires were notched by $80-90 \%$ in between the gluing points. The break was established after the system had been pumped to vacuum and cooled down to 4.2 $\mathrm{K}$, thus providing two clean surfaces. Breaking the sample causes inelastic deformations, resulting in contraction of the electrodes, which become negligible only after $2-3 \mathrm{~h}^{7}$ After this "relaxation" time, the drift of the contact (tunnel) resistance $R_{T}$ is in general well below $2 \%$ within the typical measuring time $(5-10 \mathrm{~min})$.

The contact can be brought into the tunneling regime by a differential screw with an estimated accuracy of 2-3 $\AA$. A piezodriver was used for fine tuning and measuring $I(z)$ characteristics from the common STM regime $\left(R_{T} \sim 1-10\right.$ $\mathrm{G} \Omega$ ) up to the stable one-atom point contact. The resistance value of this contact, $\sim 13 \mathrm{k} \Omega$ for $\mathrm{Al}$ and $\mathrm{Au}$ and a value twice as low for Pt (-Ir), ${ }^{4}$ corresponds to "one quantum (two quanta) of conductance" $\left(\simeq h / 2 e^{2}\right) .{ }^{9}$ Only contacts which displayed a clear "jump" to a stable one-atom point contact were examined. In that case the electrodes can be represented fairly well by two metallic slabs with a front atom on one of them. This kind of model for a tip-sample configuration has often been used in theoretical calculations describing the tunnel characteristics. ${ }^{6,10-12}$

Because of its rigid configuration, a MCB is extremely stable compared to a STM. I $(z)$ curves with negligible noise can be taken at a slow rate, so that we can observe even subtle effects due to electrode-electrode interactions. For the same reason, it also has a major drawback. In principle a MCB has no lateral scanning abilities like a STM. Recently, we managed to do limited line scanning over several atomic distances without losing stability. To this end one of the wire ends had been glued to a small shear piezo, while the other 
one was supported by a piece of insulating material. By applying a voltage to the shear piezo, the electrodes (wire ends) move laterally with respect to each other. In this way the tunnel current (or resistance) can be measured as a function of the lateral position of the electrodes with respect to each other. The interpretation of these data is a matter of discussion, and will be returned to in Sec. III.

Another disadvantage compared to a STM is the difficulty of calibrating the distance of piezovoltage ratio. For the $z$ direction (piezodriver) this ratio strongly depends on bending angle of the bending beam and the shape of the electrodes close to the contact. The magnitude of the bending angle depends on the stage at which the sample breaks. This, in turn, depends on the notch, the mounting, and the sample material. However, once the sample has been broken, the bending angle is fairly constant. Based on the geometry and material parameters of the bending beam and the sample, we estimated the deviation from linear proportionality of $z_{0} / V_{\text {piezo }}$ to be less than $0.0001 \%$ for variations of a few ten nanometers of the electrode spacing. ${ }^{7}$ In practice we estimate distances by choosing a fixed point and then calculate the distance with respect to that point by means of the following formula giving the dependence of tunnel current $(I)$ on electrode-electrode separation $(z):{ }^{13}$

$$
I(z)=A V_{b} \exp \left(B k_{0} z\right) .
$$

where $A$ and $B$ are constants, $V_{b}$ is the applied bias voltage, and $k_{0}$ is the inverse decay length of the electrode material.

As the fixed point we choose the onset of the deviation from the simple exponential in $I(z)$ curves. In our measurements this always occurs around $R_{T}=1 \mathrm{M} \Omega$. From several other papers we can conclude that this should correspond to an electrode spacing of $\sim 3 \AA .{ }^{3,4,14}$

\section{RESULTS AND DISCUSSION}

Strong adhesion forces between the electrodes become clearly visible at separations of $\sim 3 \AA .^{3}$ A plot of $R_{T}$ versus

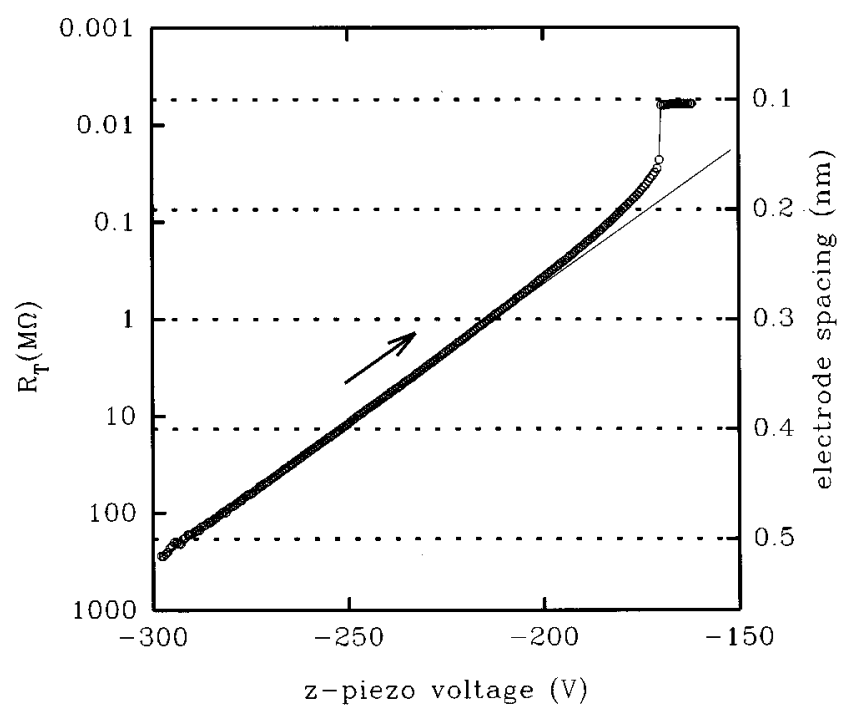

FIG. 1. The tunnel resistance vs $z$-piezo voltage. Strong adhesion forces are clearly visible. The arrow indicates the direction in which the electrodes move (in this case they approach each other). The straight (solid) line is a guide to the eye. These data were taken on $\mathrm{Pt}$, at $4.2 \mathrm{~K}$ in vacuum surroundings. The applied bias voltage was $5 \mathrm{mV}$.

$V_{\text {piezo }}$ (distance), shows a stronger than exponential dependence when the separation drops below this value. ${ }^{4}$ Figure 1 shows a clear deviation from the exponential dependence. As the distance decreases, the forces increase, reaching a critical separation at which the electrode-electrode configuration becomes mechanically unstable. Smith et al. calculated that an avalanche due to the strong adhesion would lead to contact within $100 \mathrm{fs}{ }^{15}$ This has been observed by us, and was previously reported in Refs. 3, 4, and 16, as a "jump to contact." Moreover, this instability does not depend on the stability of the instrument supporting the junction. The avalanche is inevitable, even in the very rigid MCB configu-

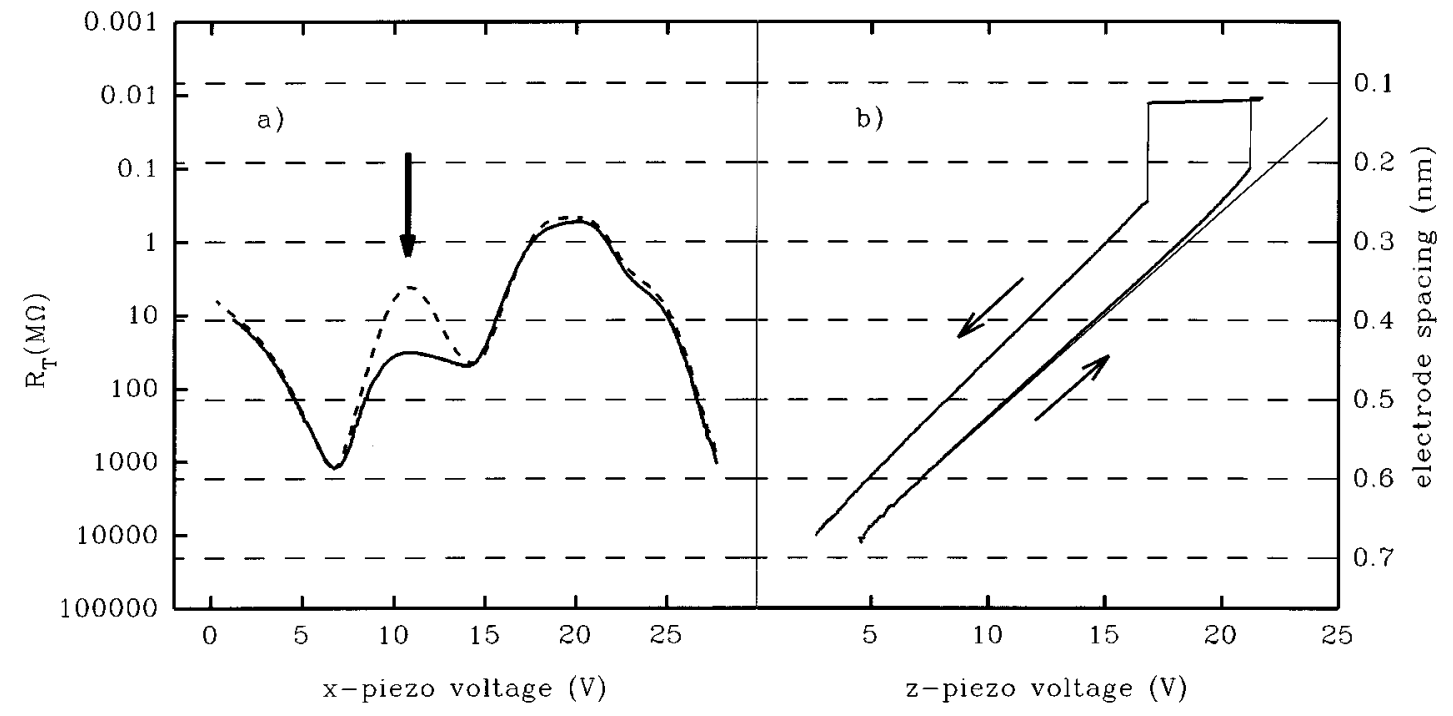

FIG. 2. The right panel (b) shows an $I(z)$ curve taken at the spot indicated by the arrow in panel (a). The left panel (a) consists of two line scans along a Au surface. The solid curve is taken before the $I(z)$ curve, and the dashed one is taken afterwards. These data clearly show the characteristics described in the text $\left(T=4.2 \mathrm{~K}\right.$, vacuum, $\left.V_{b}=10 \mathrm{mV}\right)$. 
ration. The critical separation depends on the wire material and is estimated to vary from $1.5 \AA$ (e.g., Pt) to $1.9 \AA$ (e.g., $\mathrm{Au}$ and $\mathrm{Al}$ ), in agreement with our observations. ${ }^{15,14,17}$ Based on geometrical arguments, it is expected that a tiplike (sharp) electrode is stiffer than a samplelike (flat) electrode. This means that, in STM, surface atoms of the sample will jump toward the tip. ${ }^{15}$

Using the mounted shear piezo, we measured the tunnel resistance $\left(R_{T}\right)$ as a function of lateral displacement on several materials $[\mathrm{Pt}(-\mathrm{Ir}), \mathrm{Au}$, and $\mathrm{Al}]$. The measurements presented in this paper were taken without any feedback employed. The variation in $\log \left(R_{T}\right)$ is a convolution of the topography of the two electrode surfaces, because of its linear dependence on electrode spacing $(z)$. The linearity holds for distances exceeding $\sim 3 \AA$. Below this value the interpretation is no longer valid due to the strong adhesion forces, which change the simple linear relation dramatically, as shown. If one electrode is much sharper than the opposite one, the convolution of the surfaces is that of a relatively smooth function with a delta function, like the tip and sample in STM. This is already the case in a tunnel junction consisting of two metallic slabs separated by an insulator (e.g., vacuum), in which on one of the slabs an atom is sticking out of the surface by half its radius. This led us to believe that if there is a small flat part on an otherwise rather rough electrode surface (inherent on MCB), the opposite electrode is acting like a tip in that area.

Relatively flat parts on the surface could be found on $\mathrm{Al}$, $\mathrm{Au}$, and Pt. Due to the higher relaxation rate for softer materials, it was easier to find flat parts on $\mathrm{Au}$ and $\mathrm{Al}$ than on Pt. Occasionally, we recorded line scans on $\mathrm{Au}$ and $\mathrm{Al}$ which were atomically flat over $6-8$ atomic distances. The best we found for $\mathrm{Pt}$, was a variation in height of $\sim 2$ atomic diameters over about eight atomic distances. Line scans on $\mathrm{Au}$ and $\mathrm{Al}$ are presented in Figs. 2 and 3. A line scan on Pt is shown elsewhere. ${ }^{7}$

Figure 2 is another demonstration of the presence of strong adhesion forces and the occurrence of the avalanche effect. After line scanning a certain area, indicated by the solid line in panel (a), $I(z)$ characteristics [panel (b)] were measured on the spot marked by the arrow in panel (a). As the electrodes approach each other, we again observe a stronger than exponential dependence of $R_{T}$ (or current $I_{T}$ ) on electrode separation (z), starting at $\sim 3 \AA(=1 \mathrm{M} \Omega)$. After the jump to contact, we immediately reversed the motion of the $z$-piezodriver, as to pull the electrodes apart. In spite of this quick reversion, a large hysteresis and a jump-off contact to an electrode separation of $\sim 2.5 \AA$ instead of $\sim 2 \AA$ are observed. The dashed line in the left panel shows a line scan of the same area as before, but taken after the $I(z)$ measurements. On the spot where the electrodes formed an atomic point contact, a hillock has arisen. Taking into account hysteresis in the piezomaterial of the $z$ driver is a far from sufficient explanation, because we determined it to be negligibly small, $^{7}$ and it can in no way be related to the arising of a hillock. A schematic description of the hysteresis effect and the arising of the hillock can be found in Ref. 18. In the avalanche, an atom of the flat electrode is pulled towards the sharper tiplike electrode. In retracting the electrodes a small conduction channel (a so-called neck ${ }^{18}$ ) of one atom point contact wide is maintained between the electrodes, due to the
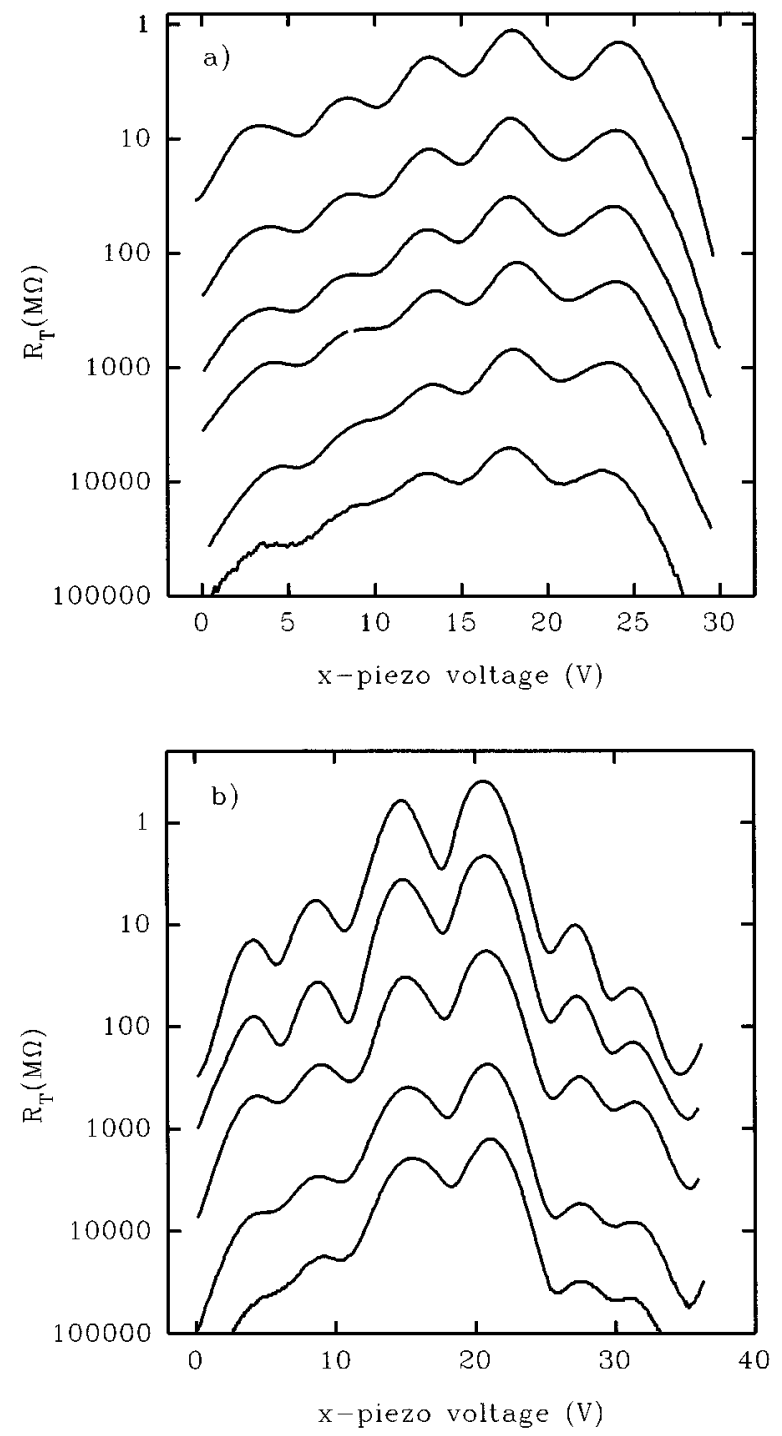

FIG. 3. Line scans taken on $\mathrm{Al}$ (a) and $\mathrm{Au}$ (b). The corrugation clearly increases as the electrode spacing decreases ( $R_{T}$ decreases). One decade in resistance corresponds to $\sim 1 \AA$, and at $1 \mathrm{M} \Omega$ the separation is $\sim 3 \AA$. To emphasize the stability of the MCB, it makes no difference if we plot all the data points or make a line plot as is done here (to reduce printing time). These measurements were taken at $4.2 \mathrm{~K}$ in a vacuum environment with a $100-\mathrm{mV}$ bias voltage.

strong adhesion. Eventually the neck breaks, leaving a hillock on the samplelike electrode. After this neck pulling, an $I(z)$ curve back and forth on the spot of the hillock will show no hysteresis, in agreement with the stiffness of two pointlike electrodes.

Figure 3 shows line scans on $\mathrm{Au}$ and $\mathrm{Al}$, taken at several values of $R_{T}$, i.e., several electrode spacings. For each line scan, the logarithmically plotted variation in $R_{T}$ can directly be interpreted as the corrugation of the metal surface, because of the earlier mentioned linear relation between $\log \left(R_{T}\right)$ and $z$. As the separation decreases, the corrugation increases, going to very large variations in height for small electrode spacings. Anomalously high corrugations on $\mathrm{Al}$ 
(Ref. 5) and Au (Ref. 19) have previously been reported. Because the lateral resolution is not affected, the changing corrugation is probably not of a geometrical nature. An enhancement in corrugation due to elastic deformation of the electrodes, which was quite successful with regard to laminar materials, was put into question by Tekman and Ciraci. ${ }^{6,10}$ They calculated the tunnel current starting from a realistic potential, by including tip-sample interactions (ion-ion and electron-ion). This yields an effective barrier $\left(\phi_{\text {eff }}\right)$, which holds for small tip-sample separations $(\leqslant 7 \AA)$, where the TH approximation ${ }^{1}$ breaks down. It is found that $\phi_{\text {eff }}$ does not collapse, so that the charge transport is carried by a tunnel current even at very small distances. The most striking feature of this effective barrier is its site dependence, which accounts for the anomalous height and distance dependence of the corrugations observed.

In Fig. 4 the corrugation versus electrode spacing has been plotted. A fit to the data results in a linear function. This is in agreement with theory and has previously been shown in experiments by Wintterlin et al. ${ }^{5}$ Some rewriting of Eq. (1) gives

$$
\Delta \xi=\frac{k_{t}-k_{h}}{k_{0}} z_{0} .
$$

$\Delta \xi$ is a corrugation, $k_{t}$ and $k_{h}$ are the apparent inverse decay lengths above a top and a hollow site, respectively, $k_{0}$ is the inverse decay length of the electrode material, and $z_{0}$ is the electrode-electrode separation. All the parameters on the right-hand side are constant except for $z_{0}$.

At $z_{0} \simeq 2.5 \AA$ a corrugation of $\sim 0.3 \AA$ is predicted for $\operatorname{Al}(111),{ }^{6}$ in agreement with previous observations. ${ }^{5}$ At $z_{0} \simeq 2 \AA$ the corrugation is predicted to invert, but due to the mechanical instability it will be difficult to observe this. In fact we have not been able to scan surfaces for separations smaller than $\sim 3 \AA$, without making contact and thereby changing the geometry of the electrodes. For $\mathrm{Al}$, a fairly good agreement with the predictions of Refs. 6 and 10, and the observations in Ref. 5, is found. It was pointed out by Schuster et al. that corrugations on Au could be as large as $0.8-1.0 \AA .{ }^{19}$ The maximum value we observed yields $\sim 0.8$ $\AA$.

\section{CONCLUSIONS}

The stability of a MCB makes this instrument suitable for detailed investigations on small variations due to mechanical

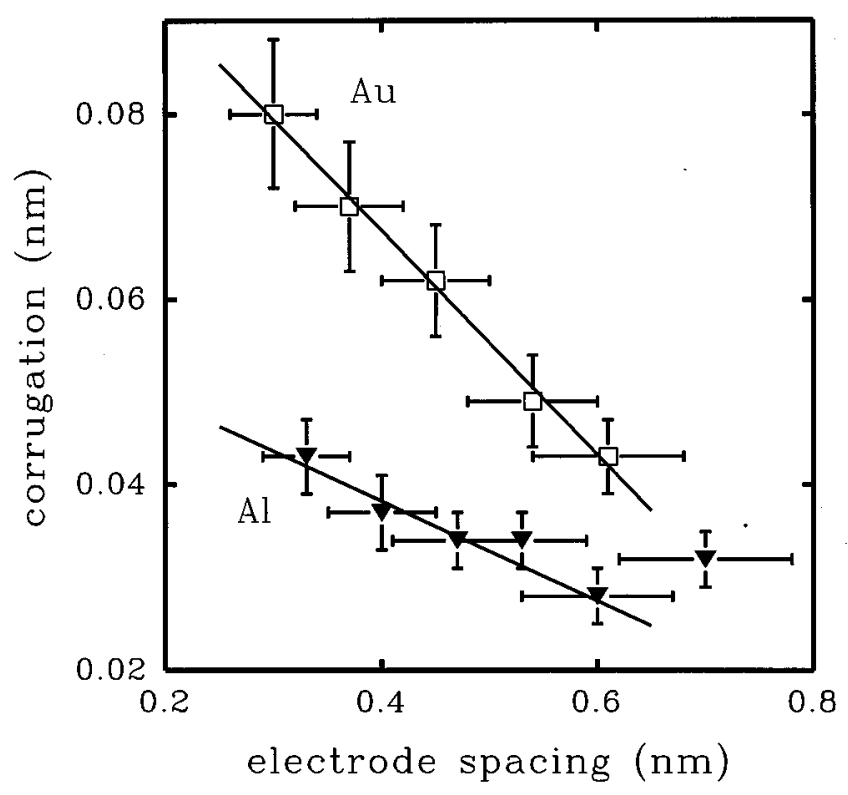

FIG. 4. The corrugation as a function of electrode spacing for $\mathrm{Al}$ and $\mathrm{Au}$. The corrugation has been determined using the highestlying atoms of each line scan in Fig. 3. The lines are a fit to the data. For Al, only the four points coinciding with the line have been included in the fitting procedure. For $\mathrm{Au}$, the line is a fit to all data points. The large errors stem from our estimation of the "fixed" point $(1 \mathrm{M} \Omega \sim 3 \AA$ ) and the uncertainty in the work function.

and electrical interactions on an atomic scale. Force effects and anomalous corrugations previously observed in STM experiments were clearly revealed by measurements in our highly stable MCB configuration. Although the MCB has only a limited scanning capability, its inherent rigidity makes it a useful complementary instrument to the STM, to study the tunneling phenomenon in detail.

\section{ACKNOWLEDGMENTS}

Part of this work was supported by the Stichting voor Fundamenteel Onderzoek der Materie (FOM) which is financially supported by the Nederlandse Organisatie voor Wetenschappelijk Onderzoek (NWO). We thank J.G.H. Hermsen, J. W. Gerritsen, and E.J.G. Boon for technical and calculational help. O.I.S. acknowledges NWO for a visitor's grant.
${ }^{1}$ J. Tersoff and D. R. Hamann, Phys. Rev. Lett. 50, 1998 (1983).

${ }^{2}$ C. J. Muller et al., Physica C 191, 485 (1992).

${ }^{3}$ U. Dürig et al., Phys. Rev. Lett. 65, 349 (1990).

${ }^{4}$ J. M. Krans et al., Phys. Rev. B 48, 14721 (1993).

${ }^{5}$ J. Wintterlin et al., Phys. Rev. Lett. 62, 59 (1989).

${ }^{6}$ E. Tekman and S. Ciraci, Phys. Rev. B 42, 1860 (1990).

${ }^{7}$ R. J. P. Keijsers et al. (unpublished).

${ }^{8}$ H. van Kempen and O. I. Shklyarevskii, Fiz. Nizk. Temp. 19, 816 (1993) [Sov. J. Low Temp. Phys. 19, 583 (1993)].

${ }^{9}$ T. N. Todorov and A. P. Sutton, Phys. Rev. Lett. 70, 2138 (1993).

${ }^{10}$ S. Ciraci, Ultramicroscopy 42-44, 16 (1992).
${ }^{11}$ G. Doyen et al., Appl. Phys. A 51, 281 (1990).

${ }^{12}$ Th. Laloyaux et al., Phys. Rev. B 47, 7508 (1993).

${ }^{13}$ L.E.C. van de Leemput, Ph.D. thesis, Nijmegen (1991).

${ }^{14}$ J. Ferrer et al., Phys. Rev. B 38, 10113 (1988).

${ }^{15}$ J. R. Smith et al., Phys. Rev. Lett. 63, 1269 (1989).

${ }^{16}$ J. K. Gimzewski and R. Möller, Phys. Rev. B 36, 1284 (1987).

${ }^{17}$ J. B. Pethica and A. P. Sutton, J. Vac. Sci. Technol. A 6, 2490 (1988).

${ }^{18}$ J. I. Pascual et al., Phys. Rev. Lett. 71, 1852 (1993).

${ }^{19}$ R. Schuster et al., Ultramicroscopy 42-44, 533 (1992). 